\title{
Photocatalytic $\alpha$-Alkylation of Amines with Alkyl Halides
}

\author{
Lingying Leng and Joseph M. Ready* \\ UT Southwestern Medical Center, 5323 Harry Hines Blvd., Dallas, Texas 75390
}

\author{
Supporting Information Placeholder
}

\begin{abstract}
Branched amines represent essential building blocks for organic synthesis. They are traditionally prepared through nucleophilic addition to imines. These methods often require highly reactive organometallic reagents and proceed under rigorous air- and moisture-free conditions. Here we describe an alternative approach that involves a net dehydrogenative coupling between alkyl bromides and amines. Mechanistically, the reaction likely involves photocatalytic generation of an $\alpha$-amino radical and a stabilized carbon-centered radical (allyl, benzyl, $\alpha$-carbonyl) followed by radical recombination. This approach offers a mild, atomeconomical, redox neutral synthesis of $\alpha$-branched amines that shows broad scope and avoids pre-metalated reagents.
\end{abstract}

Branched amines (A) are components of natural products, pharmaceutical agents, and agricultural chemicals. They are key building blocks for organic synthesis and subunits of ligands for transition metals. Traditional synthetic approaches to $\alpha$-branched amines generally rely on addition of a carbon-based nucleophile to an imine electrophile (Figure 1a). This general strategy encompasses venerable methods such as the Strecker $\left(\mathrm{CN}^{-}\right)^{1}$ and Mannich (enolate $)^{2}$ reactions. Organometallic additions can involve highly reactive Grignard or organolithium reagents or milder nucleophiles like organoboron species. ${ }^{3,4,5,6}$ Reductive amination extends this concept to hydride nucleophiles. While these traditional nucleophile/electrophile pairings have been utilized extensively, they often require highly reactive or specialized nucleophiles, which limits their generality and scalability.

Inverting the normal polarity of imine addition suggested an alternative route to $\alpha$-branched amines. In principle, an $\alpha-\mathrm{C}-\mathrm{H}$ alkylation of amines with a suitable electrophile could yield the desired products (Figure 1b). In practice, however, $N$-alkylation is expected to occur between an amine and an electrophile. Notwithstanding the facility of $\mathrm{N}$-alkylation, recent discoveries in the area of photocatalysis appeared to offer the opportunity to develop a mild, umpolung $C$-alkylation of amines. ${ }^{7}$

Pandey, Reiser and Yoon independently demonstrated that an excited state Ir or Ru photocatalyst could generate a nucleophilic $\alpha$-amino radical $\mathbf{B}$ through single-electron transfer (SET) and proton loss (Figure 1c)..$^{8,9,10}$ Subsequently, Giese addition to an electron-deficient olefin led to the $\alpha$-branched amine products. Our laboratory recently showed that this addition could be carried out regioselectively with nonsymmetrical amines, ${ }^{11}$ and the MacMillan group reported that the same $\alpha$-amino radical can engage (hetero)aryl halides to give $\alpha$-(hetero)aryl amines. ${ }^{12}$ Alternatively, several groups have demonstrated that the $\alpha$-amino radicals $(\mathbf{B})$ can be accessed in a reductive manifold from iminium ions, ${ }^{13,14,15}$ while
Rovis' group achieved similar transformations both photochemically and electrochemically. ${ }^{16,17}$

a. Traditional synthesis of $\alpha$-branched amines through addition to imines

Strecker $\left(\mathrm{CN}^{-}\right)$

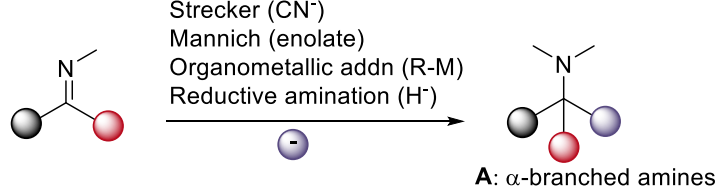

b. Alkylation of amines

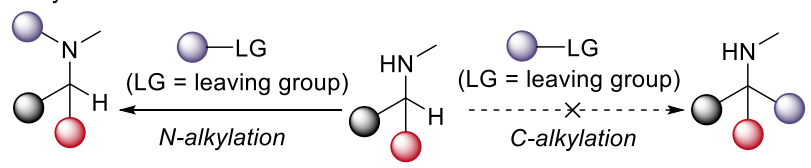

c. Photochemical $\alpha$-functionalization of amines

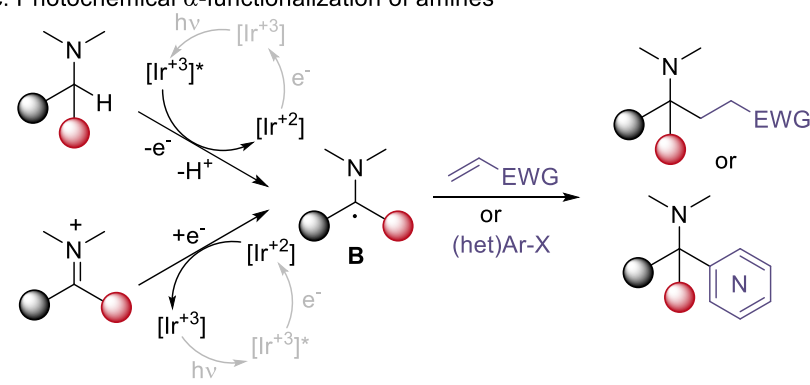

d. C-Alkylation of amines with alkyl halides (this work)
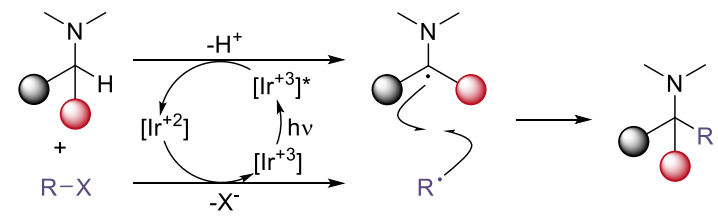

Figure 1. Synthesis of $\alpha$-branched amines.

They key step in many photocatalytic methods is the SET from an amine to the exited state $\operatorname{Ir}(\mathrm{III})^{*}$ photocatalyst to form the reduced Ir(II) species. We wondered if this $\operatorname{Ir}(\mathrm{II})$ intermediate would reduce appropriate electrophiles to form an $\alpha$-amino/alkyl radical pair (Figure 1D). Subsequent radical coupling would provide the $\alpha$-branched amine products and could represent a mild, redox-neutral C-C bond formation that avoids prior formation of reactive reagents such as those used in traditional imine addition chemistry. ${ }^{18}$ Encouragingly, $\mathrm{Xu}$ and coworkers have demonstrated the $\alpha$-alkylation of $N$-aryl glycine derivatives under photolytic conditions, ${ }^{19,20}$ while Walsh has described the alkylation and arylation of aza-allyl 


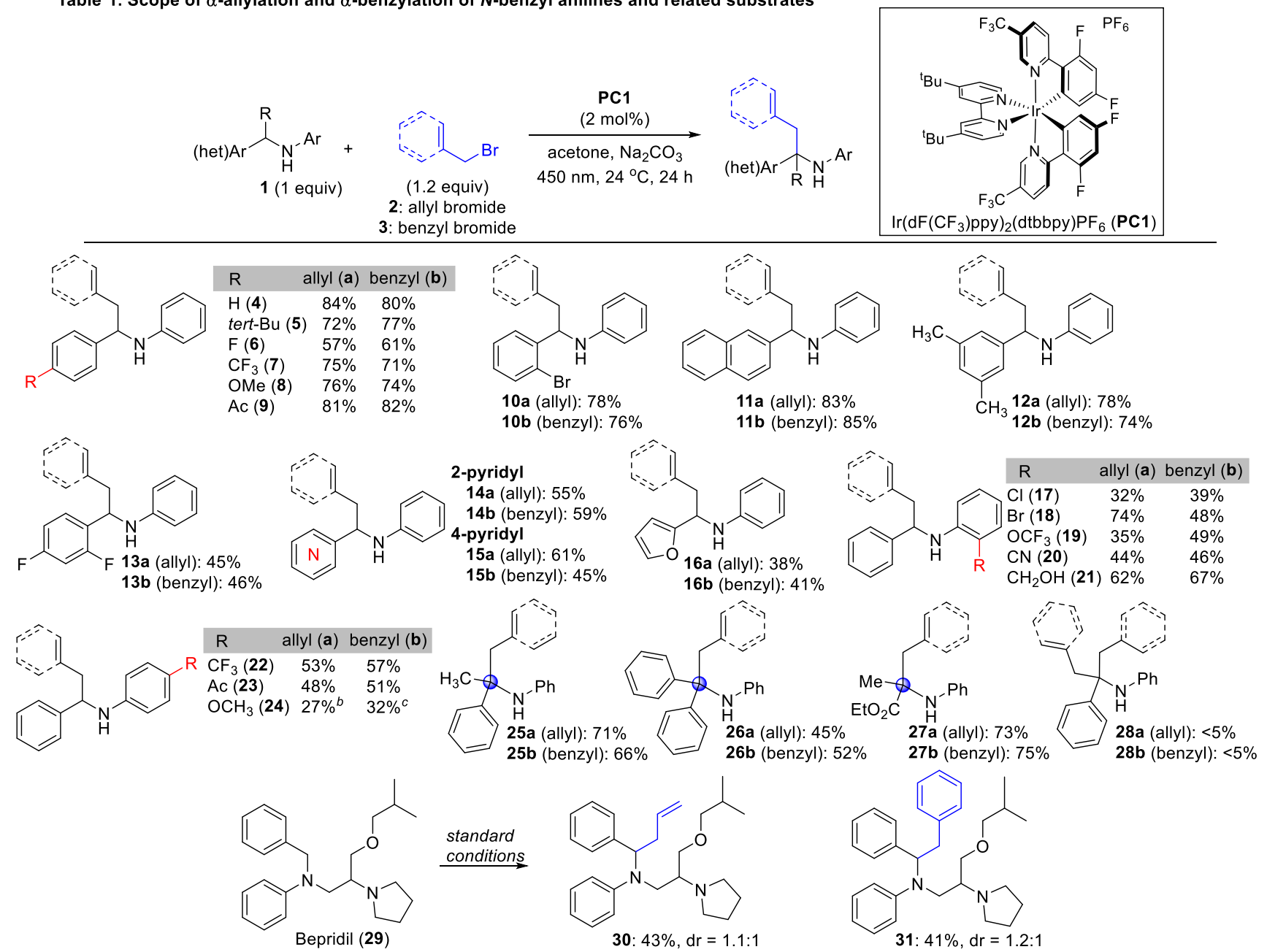

${ }^{a}$ Isolated yields. ${ }^{b} \mathrm{An}$ additional $32 \%$ of the $\mathrm{N}$-allyl isomer was isolated. ${ }^{c} \mathrm{An}$ additional $37 \%$ of the $\mathrm{N}$-Bn isomer was isolated.

anions in a thermal process that likely involves radical-radical coupling. ${ }^{21}$ Here we show the successful realization of a mild $\alpha$-alkylation of amines that involves a redox-neutral coupling with alkyl bromides.

$\alpha$-Allylation and benzylation of amines. Reaction development focused on $N$-benzyl aniline (1a) coupling with allyl (2) or benzyl (3) bromide. A brief survey of reaction parameters identified the conditions outlined in Table 1 (for reaction optimization see Supplementary Table S1). Photocatalyst PC1 $\left[\operatorname{Ir}\left(\mathrm{dF}\left(\mathrm{CF}_{3}\right) \text { ppy }\right)_{2}(\mathrm{dtbbpy})\left(\mathrm{PF}_{6}\right)\right]$ proved optimal for the $C$-alkylation, and the reactions proceeded at room temperature in acetone in the presence of a mild base $\left(\mathrm{Na}_{2} \mathrm{CO}_{2}\right)$ under $450 \mathrm{~nm}$ light. The major side product in other solvent/base/catalyst combinations was the $\mathrm{N}$-alkylated isomer. Similarly, in the absence of base or photocatalyst, the $\mathrm{N}$-alkylated isomer was formed prominently or exclusively.

The reaction tolerated electron-rich and electron-deficient $\mathrm{N}$ benzyl groups, with yields for allylation generally paralleling yields for benzylation (4 -9). Mono- and di-substitution was accommodated at all positions on existing $N$-benzyl ring (10-13). No reduction of aryl halides or a methyl ketone (9) was observed, while oxidation at methoxy ether and benzylic methyl groups was also avoided. Heteroaryl rings including pyridine $(\mathbf{1 4}, \mathbf{1 5})$ and furan rings could be introduced on the substrate successfully, although the attenuated yield in the case of furan $\mathbf{1 6}$ could implicate oxidation of that electron-rich ring over the course of the reaction.
The $N$-phenyl ring could be substituted with electron-withdrawing groups while maintaining synthetically useful yields $(\mathbf{1 7}-\mathbf{2 3})$. By contrast, electron-rich $N$-aryl rings ring provided complex mixtures, likely arising from oxidation of the aniline and $\mathrm{N}$-alkylation. For example, an aminophenol derivative yielded modest yields of the $C$-allylated (24a) and -benzylated products (24b). Low mass balances and $N$-alkylation was observed in both cases.

Some $\alpha$-quaternary amines were accessible using the photocatalytic alkylation. In detail, a substrate bearing a phenyl and methyl group adjacent to the amine could be allylated or benzylated (25a, b) successfully. Similarly, benzhydryl aniline provided synthetically useful yields of the allylated and benzylated products (26a, b). Extending the chemistry to a glycine derivative provided quaternary $\alpha$-aminoesters $\mathbf{2 7} \mathbf{a}$ and $\mathbf{2 7} \mathbf{b}$ in good yield. However, only trace quantities of di-allylated or di-benzylated products were observed (28) in the alkylation of $N$-benzyl aniline, even when excess amounts of the bromide were used. We interpret this outcome to indicate that $\alpha$-quaternary amines can be synthesized if one of the $\alpha$-substituents is small (e.g., methyl) or able to stabilize a radical (e.g., phenyl, ester).

As an example of potential opportunities in medicinal chemistry, the calcium blocker bepridil (29) was benzylated and allylated without complications arising from the tertiary amine $(\mathbf{3 0}, \mathbf{3 1})$.

Scope of alkyl bromides. The $\alpha$-alkylation proved relatively insensitive to electronic or steric differences among benzylic bromides (Table 2a). Electron-withdrawing (32-35) and electron-donating (36) groups on the electrophile had little impact on yield. 
<smiles>c1ccc(N2CCc3ccccc3C2)cc1</smiles><smiles>[R]C1c2ccccc2CCN1c1ccccc1</smiles>
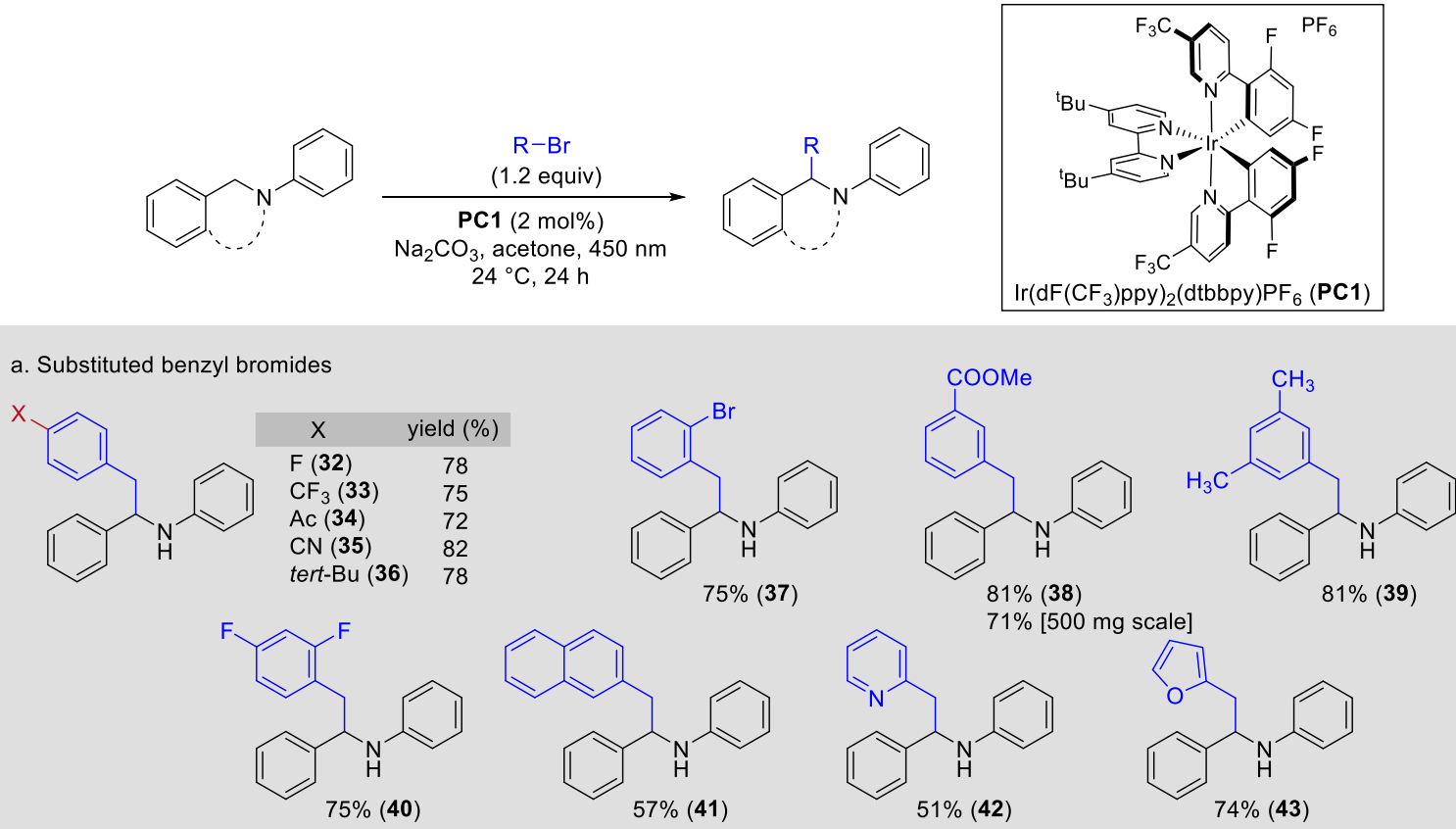

$71 \%$ [500 mg scale]
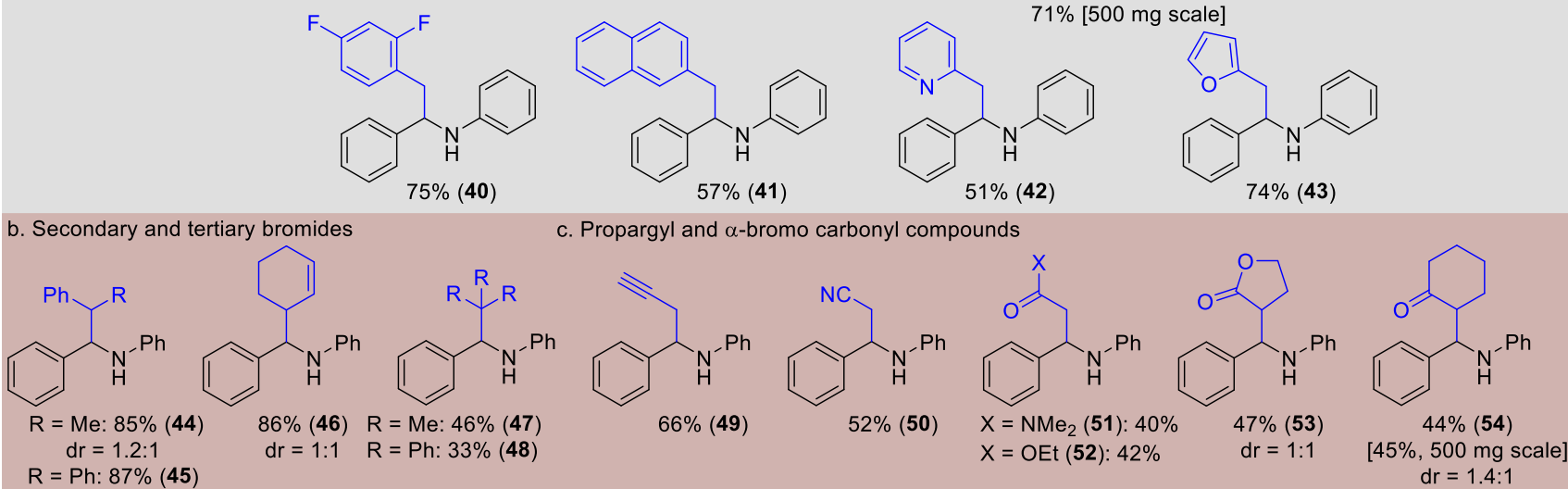

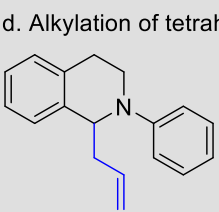

$80 \%(\mathbf{5 5})$

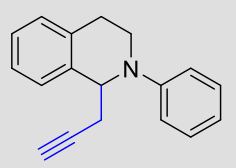

$78 \%(56)$
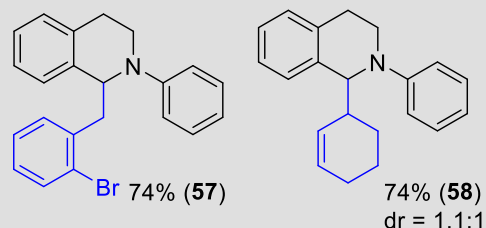
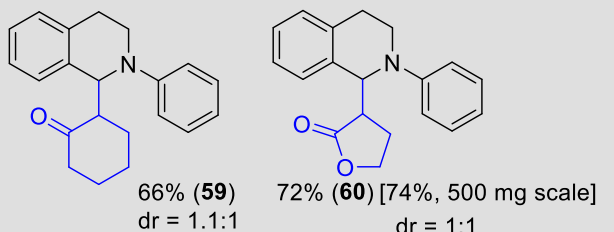

${ }^{a}$ Isolated yields. ${ }^{b}$ Reactions in acetonitrile

Likewise, substitution was accommodated at the ortho (37) and meta positions (38), and disubstituted benzyl bromides were successful coupling partners (39-40). A naphthalene (41), pyridine (42) and furan (43) were introduced successfully under the standard conditions as well. As shown in Table $2 \mathrm{~b}$, secondary benzylic (44, 45) or allylic (46) bromides provided the $\alpha$-branched amines in excellent yield. Even trityl and tert-butyl bromides $(\mathbf{4 7}, 48)$ were competent reaction partners, albeit in reduced yield.

Several additional classes of activated alkyl bromides could participate in the $\alpha$-alkylation (Table 2c). For example, propargyl bromide yielded the terminal alkyne 49 without formation of detectable levels of the corresponding allene. A series of $\alpha$-bromo carbonyl and related compounds provided amino-nitrile (50), aminoamide (51), amino-ester $(\mathbf{5 2}, \mathbf{5 3})$ and amino-ketone (54) products. Separately, tetrahydroisoquinoline represented an excellent substrate, reacting cleanly with several alkyl bromides (55-60). Complete regioselectivity was observed, and the reaction accommodated allylic, propargylic and benzylic bromides as well as $\alpha$ bromo ketones and lactones. With regard to current limitations, unactivated secondary or primary alkyl bromides (e.g., $i-\mathrm{PrBr}, n$ $\mathrm{PrBr}$ ) do not react under these conditions. Allyl chloride provides the $\alpha$-branched product in reduced yield whereas the iodide leads to exclusive $N$-alkylation (see Supplementary Table 1). Finally, the reaction performed similarly on a $\sim 30 \mathrm{mg}$ scale and $500 \mathrm{mg}$ scale as demonstrated by several examples $(\mathbf{3 8}, \mathbf{5 4}, \mathbf{6 0})$.

Alkylation with acetone. Introduction of the smallest ketone, acetone, would require bromoacetone, which is a controlled substance and a highly toxic lachrymator. As an alternative, we dis-

Table 3. $\alpha$-Alkylation of amines with in situ generated bromoacetone

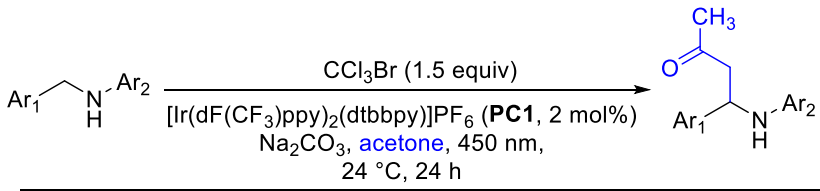
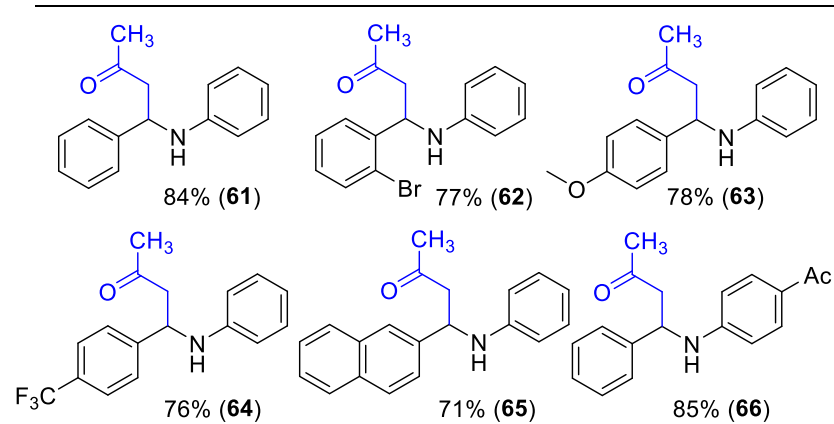


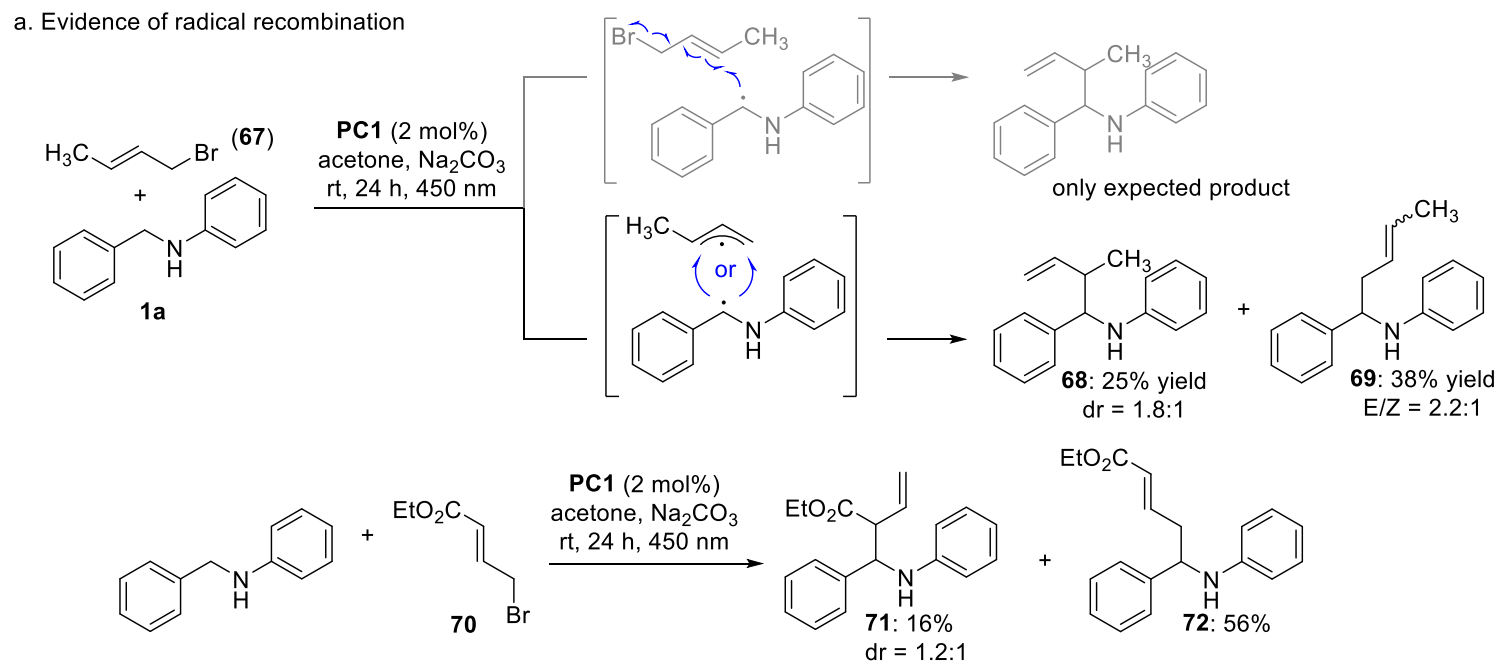

b. Evidence of $\alpha$-amino radical formation<smiles>c1ccc(CNc2ccccc2)cc1</smiles>

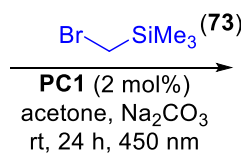

rt, $24 \mathrm{~h}, 450 \mathrm{~nm}$

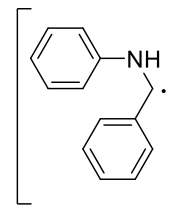

.

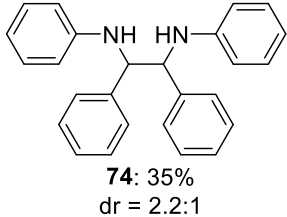

$74: 35 \%$
$d r=2.2: 1$
Figure 2. Mechanistic studies.

covered that bromotrichloromethane can effect the dehydrogenative coupling between benzylic amines and acetone (Table 3). Several substituted $N$-benzyl anilines were found to react cleanly and provide the $\alpha$-amino ketone products in $71-85 \%$ yields. Under these conditions, bromoacetone is likely generated in situ and participates analogously to other alkyl bromides, providing a safer alternative than using bromoacetone directly. ${ }^{22}$

Mechanistic considerations. Existing data supports the mechanism outlined in Figure 1d. Irradiation of PC1 generates an excited state $[\operatorname{Ir}(\mathrm{III})]^{*}$ complex that acts as an oxidant $\left(\mathrm{E}_{1 / 2}{ }^{\mathrm{ox}}=1.21 \mathrm{~V}\right.$ vs $\left.\mathrm{SCE} ; \mathrm{Ir}^{+3 *} / \mathrm{Ir}^{+2}\right)$ to accept an electron from an amine (BnNHPh $\mathrm{E}_{\mathrm{p} / 2}$ $=0.89 \mathrm{~V}) \cdot{ }^{23,24}$ Loss of proton alpha to the amine generates a carbonbased radical intermediate. Separately, the reduced $\operatorname{Ir}(\mathrm{II})$ species $\left(\mathrm{E}_{1 / 2}{ }^{\mathrm{red}}=-1.37 \mathrm{vs} \mathrm{SCE} ; \mathrm{Ir}^{+2} / \mathrm{Ir}^{+3}\right)$ could transfer an electron to the alkyl bromide $\left(\mathrm{BnBr}: \mathrm{E}^{0}=-0.51\right.$; ethyl bromoacetate: $\mathrm{E}^{0}=-0.78$; bromoacetonitrile: $\left.\mathrm{E}^{0}=-0.60\right) \cdot{ }^{25}$ Loss of bromide ion would yield a second, C-based radical, which could combine with the $\alpha$-amino radical formed through oxidation.

An alternative mechanism for the allylation reactions could involve conjugate $\mathrm{S}_{\mathrm{H}} 2$ ' substitution of the bromide (Figure $2 \mathrm{a}$ ). To investigate this possibility, we tested crotyl bromide (67) in the reaction. Radical addition to the olefin would be expected to give a single isomeric product. By contrast, radical combination could occur at either termini of the allylic radical to give a mixture of the branched (68) and linear product (69). The latter outcome was observed experimentally $(\mathbf{6 8 : 6 9}=1: 1.5)$. Similar results were obtained with allylic bromide 70. Finally, additional evidence for the intermediacy of $\alpha$-amino radicals came from a failed attempt to couple with (bromomethyl)trimethylsilane (73, Figure $2 \mathrm{~b})$. In this case, the diamine $\mathbf{7 4}$ was obtained, indicative of a radical homocoupling.

Traditional syntheses of $\alpha$-branched amines have relied on addition of reactive carbon (or hydride) nucleophiles to preformed imines. Recent advances in photocatalysis have allowed inversion of this normal polarity to facilitate the redox neutral $\alpha$-alkylation of an amine with alkyl bromides. The upshot of this approach is a mild synthesis of $\alpha$-branched amines that avoids reactive organometallic reagents and displays a broad scope. We anticipate that this method will find use in the synthesis of medicinally relevant small molecules, natural products, and other valuable amines.

\section{ASSOCIATED CONTENT}

\section{Supporting Information}

Supplementary table, experimental methods, characterization data, spectra.

\section{AUTHOR INFORMATION}

\section{Corresponding Author}

Joseph.ready@utsouthwestern.edu

\section{Notes}

The authors declare no competing financial interests.

\section{ACKNOWLEDGMENT}

Funding provided by the Welch Foundation (I-1612) and the American Chemical Society-Petroleum Research Fund (58264-ND1).

\section{REFERENCES}


(1) Yet, L. Recent developments in catalytic asymmetric Strecker-type reactions. Angew. Chem. Int. Ed. 2001, 40, 875-877.

(2) Arend, M.; Westermann, B.; Risch, N. Modern variants of the Mannich reaction. Angew. Chem. Int. Ed. 1998, 37, 1044-1070.

(3) Robak, M. T.; Herbage, M. A.; Ellman, J. A. Synthesis and applications of tert-butanesulfinamide. Chem. Rev. 2010, 110, 3600-3740.

(4) Wu, P.; Givskov, M.; Nielsen, T. E. Reactivity and synthetic applications of multicomponent petasis reactions. Chem. Rev. 2019, 119, 1124511290

(5) Kobayashi, S.; Ishitani, H. Catalytic enantioselective addition to imines. Chem. Rev. 1999, 99, 1069-1094.

(6) Kumar, R.; Flodén, N. J.; Whitehurst, W. G.; Gaunt, M. J. A general carbonyl alkylative amination for tertiary amine synthesis. Nature $\mathbf{2 0 2 0}$ $581,415-420$

(7) Beatty, J. W.; Stephenson, C. R. J. Amine functionalization via oxidative photoredox catalysis: Methodology development and complex molecule synthesis. Accts. Chem. Res. 2015, 48, 1474-1484.

(8) Kohls, P.; Jadhav, D.; Pandey, G.; Reiser, O. Visible light photoredox catalysis: Generation and addition of $N$-aryltetrahydroisoquinoline-derived $\alpha$-amino radicals to Michael acceptors. Org. Lett. 2012, 14, 672-675.

(9) Ruiz Espelt, L.; Wiensch, E. M.; Yoon, T. P. Brønsted acid cocatalysts in photocatalytic radical addition of $\alpha$-amino $\mathrm{C}-\mathrm{H}$ bonds across $\mathrm{Mi}$ chael acceptors. J. Org. Chem. 2013, 78, 4107-4114.

(10) See also: McManus, J. B.; Onuska, N. P. R.; Nicewicz, D. A. Generation and alkylation of $\alpha$-carbamyl radicals via organic photoredox catalysis. J. Am. Chem. Soc. 2018, 140, 9056-9060.

(11) Leng, L.; Fu, Y.; Liu, P.; Ready, J. M. Regioselective, photocatalytic $\alpha$-functionalization of amines. J. Am. Chem. Soc. 2020, 142, 1197211977.

(12) Prier, C. K.; MacMillan, D. W. C. Amine $\alpha$-heteroarylation via photoredox catalysis: A homolytic aromatic substitution pathway. Chem. Sci. 2014, 5, 4173-4178.

(13) Fuentes de Arriba, A. L.; Urbitsch, F.; Dixon, D. J. Umpolung synthesis of branched $\alpha$-functionalized amines from imines via photocatalytic three-component reductive coupling reactions. Chemical Commun. 2016, $52,14434-14437$

(14) Lee, K. N.; Lei, Z.; Ngai, M.-Y. $\beta$-Selective reductive coupling of alkenylpyridines with aldehydes and imines via synergistic Lewis acid/photoredox catalysis. J. Am. Chem. Soc. 2017, 139, 5003-5006.
(15) Trowbridge, A.; Reich, D.; Gaunt, M. J. Multicomponent synthesis of tertiary alkylamines by photocatalytic olefin-hydroaminoalkylation. $\mathrm{Na}$ ture 2018, 561, 522-527.

(16) Nicastri, M. C.; Lehnherr, D.; Lam, Y.-h.; DiRocco, D. A.; Rovis, $\mathrm{T}$. Synthesis of sterically hindered primary amines by concurrent tandem photoredox catalysis. J. Am. Chem. Soc. 2020, 142, 987-998.

(17) Lehnherr, D.; Lam, Y.-h.; Nicastri, M. C.; Liu, J.; Newman, J. A.; Regalado, E. L.; DiRocco, D. A.; Rovis, T. Electrochemical synthesis of hindered primary and secondary amines via proton-coupled electron transfer. J. Am. Chem. Soc. 2020, 142, 468-478

(18) Xie, J.; Jin, H.; Hashmi, A. S. K. The recent achievements of redoxneutral radical $\mathrm{C}-\mathrm{Cc}$ cross-coupling enabled by visible-light. Chem. Soc. Rev. 2017, 46, 5193-5203.

(19) Wang, C.; Guo, M.; Qi, R.; Shang, Q.; Liu, Q.; Wang, S.; Zhao, L.; Wang, R.; Xu, Z. Visible-light-driven, copper-catalyzed decarboxylative $\mathrm{C}$ (sp3)-H alkylation of glycine and peptides. Angew. Chem. Int. Ed. 2018, $57,15841-15846$

(20) Wang, C.; Qi, R.; Xue, H.; Shen, Y.; Chang, M.; Chen, Y.; Wang, $\mathrm{R}$.; Xu, Z. Visible-light-promoted C(sp3)-H alkylation by intermolecular charge transfer: Preparation of unnatural $\alpha$-amino acids and late-stage modification of peptides. Angew. Chem. Int. Ed. 2020, 59, 7461-7466.

(21) Li, M.; Berritt, S.; Matuszewski, L.; Deng, G.; Pascual-Escudero, A.; Panetti, G. B.; Poznik, M.; Yang, X.; Chruma, J. J.; Walsh, P. J. Transition-metal-free radical $\mathrm{C}(\mathrm{sp} 3)-\mathrm{C}(\mathrm{sp} 2)$ and $\mathrm{C}(\mathrm{sp} 3)-\mathrm{C}(\mathrm{sp} 3)$ coupling enabled by 2-azaallyls as super-electron-donors and coupling-partners. J. Am. Chem. Soc. 2017, 139, 16327-16333.

(22) Huo, H.; Wang, C.; Harms, K.; Meggers, E. Enantioselective, catalytic trichloromethylation through visible-light-activated photoredox catalysis with a chiral iridium complex. J. Am. Chem. Soc. 2015, 137, 95519554.

(23) Speckmeier, E.; Fischer, T. G.; Zeitler, K. A toolbox approach to construct broadly applicable metal-free catalysts for photoredox chemistry: Deliberate tuning of redox potentials and importance of halogens in donoracceptor cyanoarenes. J. Am. Chem. Soc. 2018, 140, 15353-15365.

(24) Roth, H. G.; Romero, N. A.; Nicewicz, D. A. Experimental and calculated electrochemical potentials of common organic molecules for applications to single-electron redox chemistry. Synlett 2016, 27, 714-723.

(25) Isse, A. A.; Lin, C. Y.; Coote, M. L.; Gennaro, A. Estimation of standard reduction potentials of halogen atoms and alkyl halides. J. Phys. Chem. B 2011, 115, 678-684 


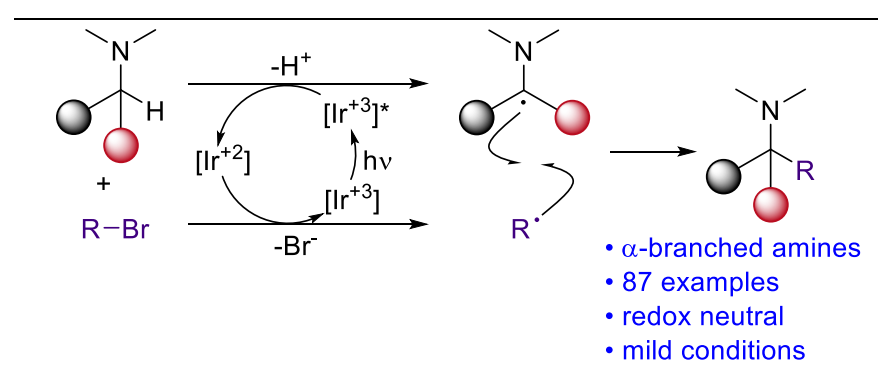

Insert Table of Contents artwork here 